04

\title{
Экспериментальное обнаружение признаков кулоновского взрыва в плазме микропинча
}

\author{
() А.Н. Долгов ${ }^{1}$, Н.А. Клячин ${ }^{2}$, Д.Е. Прохорович ${ }^{1,2}$ \\ ${ }^{1}$ Всероссийский научно-исследовательский институт автоматики им. Н.Л. Духова, Москва, Россия \\ ${ }^{2}$ Национальный исследовательский ядерный университет „МИФИ“, Москва, Россия \\ E-mail: alnikdolgov@mail.ru
}

Поступило в Редакцию 4 декабря 2018г.

В окончательной редакции 17 декабря 2018 г.

Принято к публикации 19 декабря 2018г.

\begin{abstract}
В низкоиндуктивной сильноточной вакуумной искре зафиксирован поток ионов с энергиями около $3 \mathrm{MeV}$, распространяющийся в направлении, перпендикулярном оси симметрии разряда. Оценки показывают, что зарегистрированные энергии ионов вполне соответствуют величине, достижимой в результате „кулоновского взрыва“ перетяжки плазменного канала тока разряда на стадии, предшествующей радиационному сжатию.
\end{abstract}

DOI: 10.21883/PJTF.2019.06.47502.17625

В настоящей работе авторы представляют результаты выполненных экспериментов, свидетельствующих, по их мнению, о реализации механизма „кулоновского взрыва“ вследствие убегания ускоренных в осевом направлении электронов и образования области плазмы с избыточным положительным зарядом как возможной причине формирования потока высокоэнергетических ионов в сильноточном $Z$-пинче [1].

Объектом исследований являлись корпускулярные потоки, эмитируемые из плазмы разряда низкоиндуктивной сильноточной вакуумной искры, горящего в парах материалов электродной системы (рис. 1). В качестве материала электродов использовалась сталь марки Ст. 3. Достигаемая сила тока $140 \mathrm{kA}$, время нарастания тока $1.5 \mu \mathrm{s}$ [2]. Отличительная черта схемы наших экспериментов состоит в том, что внутренний электрод является катодом. Следствием является распространение потока ускоренных в плазме разряда электронов за пределы электродной системы в осевом направлении (рис. 2), что позволяет осуществить прямую регистрацию электронного потока. Результаты прямой регистрации корпускулярной эмиссии с помощью коллектора со смещенным потенциалом $\varphi_{b}=-60 \mathrm{~V}$, размещенного на оси симметрии, но за пределами электродной системы, дали возможность оценить количество ускоренных и эмитированных из плазмы разряда электронов, след которых наблюдается в плазме разряда на рентгеновской обскурограмме, величиной $N_{e} \approx 10^{12}$. По сигналу с радиально расположенного коллектора при потенциале смещения $\varphi_{b}=+40 \mathrm{~V}$ удалось зафиксировать группу ионов железа с энергией $\varepsilon_{i} \approx 3 \mathrm{MeV}$ в соответствии с временем пролета в дрейфовом пространстве. В качестве реперных временны́х меток использовались следующие: на осциллограммах сигнала с коллектора ионов - фотопик, возникающий под действием ВУФ-излучения разряда; на осциллограмме сигнала с пояса Роговского „особенность“ тока разряда.
Дополнительно была использована методика регистрации потока ионов в радиальном направлении с помощью трекового детектора - прозрачной полимерной пленки $C R-39$. На поверхность пленки направлялся по нормали к ней коллимированный пучок ионов из разряда. Между детектором и коллиматором располагалась область поперечного по отношению к направлению распространения ионного пучка и близкого к однородному по своей пространственной структуре магнитного поля индукцией $B=0.05$ Т и протяженностью $L=8 \mathrm{~cm}$, созданного парой съемных постоянных плоских магнитов. Измерение величины смещения ионного пучка в плоскости трекового детектора под действием приложенного магнитного поля позволило оценить величину энергии наиболее высокоэнергетических регистрируемых ионов. Подсчет плотности треков на поверхности детектора, оставленных регистрируемыми ионами, после их визуализации путем травления детектора в водном растворе щелочи вплоть до проявления максимального количества треков дал возможность измерить флюенс ионов.

Наблюдаемое на описанном выше трековом детекторе смещение наиболее высокоэнергетической компоненты ионной эмиссии из разряда $s=1-1.5 \mathrm{~mm}$ (рис. 3) соответствует ожидаемой величине

$$
s \approx L^{2} / 2 r_{L i}
$$

при выполнении условия $s \ll r_{\mathrm{L} i}, \quad$ где $r_{\mathrm{L} i}=\left(2 M_{i} \varepsilon_{i}\right)^{1 / 2} /(Z e B) \quad$ - ларморовский радиус для ионов массой $M_{i}$, при допущении, что заряд ионов составляет $Z \approx 10$. Измерение плотности треков на детекторе и полного заряда регистрируемых коллектором высокоэнергетических ионов с учетом их зарядности в предположении изотропности разлета частиц в пределах телесного угла $4 \pi$ стерадиан дало оценку полного числа высокоэнергетических ионов на уровне $N_{i} \approx 10^{9}-10^{10}$. 


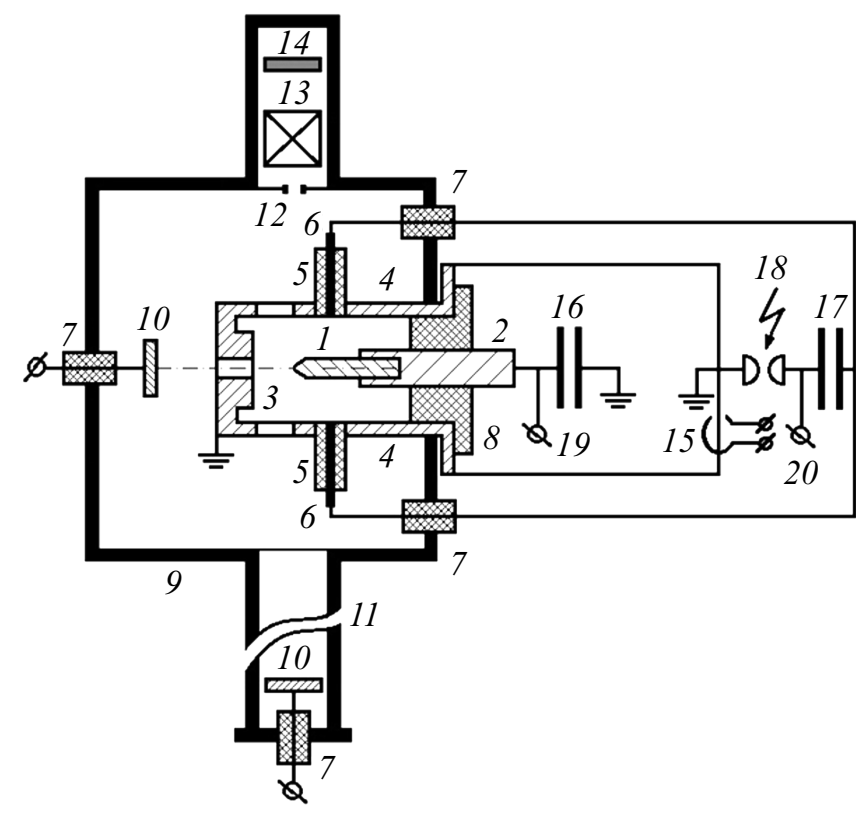

Рис. 1. Схема эксперимента. 1 - внутренний электрод, 2 - внутренний токовод, 3 - внешний электрод, 4 внешний токовод, 5 - диэлектрическая (фторопласт) втулка, 6 - поджигающий электрод, 7 - проходной изолятор, $8-$ разделительный изолятор, 9 - вакуумная камера, $10-$ коллектор заряженных частиц со смещенным потенциалом, 11 - пролетный тракт, 12 - магнитный экран со щелевой диафрагмой, 13 - параллельные плоские магниты, 14 трековый детектор, 15 - пояс Роговского, 16 - конденсаторная батарея в цепи основного разряда, 17 - конденсаторная батарея в цепи поджига, 18 - управляемый разрядник, 19, 20 - подключение источников высокого напряжения.

Оценим приобретаемую таким количеством ионов энергию $\varepsilon_{i}$ на частицу

$$
\varepsilon_{i} N_{i} \approx\left(e N_{e}^{(a c c)}\right)^{2} /\left(4 \pi \varepsilon_{0} r\right)=\left(e^{2} Z^{2} N_{i}^{2}\right) /\left(4 \pi \varepsilon_{0} r\right),
$$

где $\varepsilon_{0}-$ диэлектрическая постоянная, $r-$ радиус перетяжки, $Z$ - средний заряд иона, $N_{e}^{(a c c)}-$ количество убегающих электронов. Процесс формирования микропинча происходит в две стадии, т.е. канал тока претерпевает сначала первое магнитогидродинамическое сжатие, обусловленное вытеканием вещества из области перетяжки, до радиуса примерно $r^{(1)} \sim 10^{-4} \mathrm{~m}$, а вслед за тем второе радиационное сжатие, обусловленное излучательными потерями энергии, до радиуса порядка $r^{(2)} \sim 10^{-5} \mathrm{~m}$. На стадии первого сжатия $Z^{(1)} \approx 6$, на стадии второго сжатия $Z^{(2)} \approx 20 \quad[3,4]$. Численные оценки в случае „кулоновского взрыва“ на стадии первого сжатия дают величину $\varepsilon_{i}^{(1)} \sim 1 \mathrm{MeV}$, а для случая „кулоновского взрыва“ на стадии второго сжатия $-\varepsilon_{i}^{(2)} \sim 100 \mathrm{MeV}-1 \mathrm{GeV}$. Времяпролетная методика регистрации частиц не позволяла нам зафиксировать частицы с энергиями, соответствующими ситуации, когда „кулоновский взрыв“ осуществляется во втором сжатии. В использованном нами полимерном детекторе треки частиц с энергиями $\varepsilon_{i}^{(1)} \sim 1 \mathrm{MeV}$ и $\varepsilon_{i}^{(2)} \sim 100 \mathrm{MeV}-1 \mathrm{GeV}$ после выполнения операции травления должны выглядеть существенно различным образом. В ряде экспериментов поверхность трекового детектора специально располагалась под углом $45^{\circ}$ относительно направления падающего на нее потока частиц. Протяженные треки, отвечающие ионам с энергией $\varepsilon_{i}^{(2)} \sim 100 \mathrm{MeV}-1 \mathrm{GeV}$, не наблюдались.

Регистрация энергий ионов двумя независимыми методами дает основания считать их величину достоверной. Кроме того, существуют и другие свидетельства эмиссии высокоэнергетических ионов из плазмы пинчевых разрядов.

Ранее сообщалось о регистрации эмитируемых из плазмы пинчевого разряда, содержащего тяжелые элементы, при достигаемой силе тока на уровне сотен килоампер ионов мегаэлектронвольтных энергий. В [5] приведены данные о регистрации ионов алюминия мегаэлектронвольтных энергий из плазменного фокуса, в состав рабочего газа которого входил помимо дейтерия ксенон, а в центре медного анода там, где происходит сжатие плазменного канала тока, имелась вставка из алюминия. Авторы [6] реализовали пинчевой разряд в струе аргона, инжектируемой в вакуум, и обнаружили эмиссию ионов аргона с мегаэлектронвольтными энергиями. В [7] сообщается о том, что в вакуумной искре были обнаружены ионы углерода, ускоренные до энергий 15-20 MeV.

Пинчевые разряды в среде тяжелых элементов с уровнем достигаемых токов сто и более килоампер (при скорости нарастания тока порядка $10^{11} \mathrm{~A} / \mathrm{s}$ и более) объединяет некоторое общее свойство: в них наблюдается рождение так называемых горячих точек, т.е. сгустков плотной горячей плазмы микронных размеров, являющихся ярким источником мягкого рентгеновского излучения. Подобные объекты независимо от способа их реализации ( $X$-пинч, вакуумный разряд в струе газа или плазменном факеле, плазменный фокус) являются продуктом развития процесса пинчевания в условиях высокого уровня лучистых потерь энергии [3,8-14], а процесс их рождения получил название микропинчевания.

Эмиссия надтепловых заряженных частиц, в том числе ионов, - характерное свойство этих разрядов. Было предложено множество механизмов, которые могли бы отвечать за данное явление, однако предлагаемые модели, как правило, рассматривают ускорение ионов в отрыве от всей совокупности наблюдаемых физических явлений, поэтому происходит неувязка этих предположений с экспериментальными данными. По мнению авторов обзора [15], основанному на тщательном анализе обширного исследовательского материала, ускорение ионов до столь высоких энергий, как наблюдаемые в экспериментах, - результат сильного коллективного взаимодействия электронного пучка с ионами плазмы. 


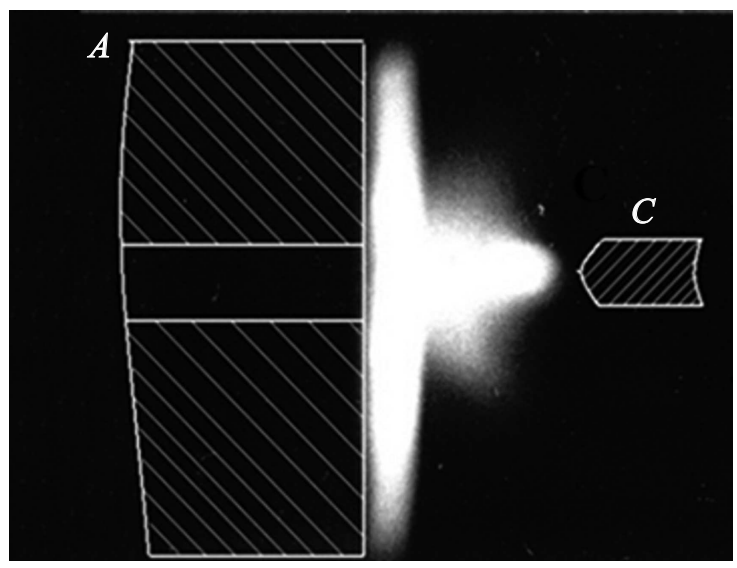

Рис. 2. Обскурограмма разряда низкоиндуктивной сильноточной вакуумной искры. Поток электронов, ускоренных в плазме разряда, распространяется в направлении анода и далее в пределах апертуры осевого отверстия в аноде за пределы электродной системы.

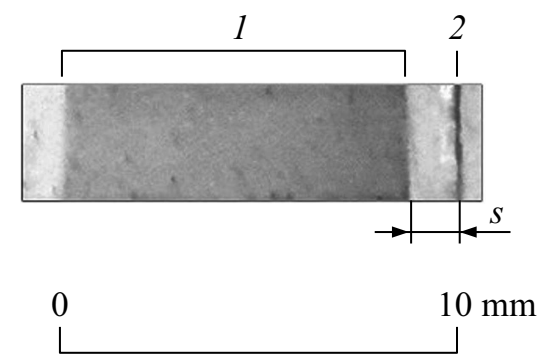

Рис. 3. След пучка высокоэнергетических ионов на трековом детекторе в присутствии магнитного поля (обозначен цифрой 1). Черта справа - метка границы следа ионного пучка на детекторе в отсутствие магнитного поля (обозначена цифрой 2).

В качестве такового может выступать так называемый „кулоновский взрыв“. По мнению авторов аналитического исследования [16], в экспериментах, описанных в [17-19] и посвященных исследованию $X$-пинчей, именно „кулоновский взрыв“ микропинча и наблюдался.

Исследователи практически без исключений регистрируют параметры ионной эмиссии только в направлении оси разряда, так как это соответствует обычно принимаемым во внимание механизмам ускорения ионов в разряде. Мы же зарегистрировали эмиссию ионов мегаэлектронвольтных энергий в радиальном направлении, что дает нам основания проанализировать полученные результаты с точки зрения осуществимости механизма „кулоновского взрыва“.

Оценим число ионов $N_{i}^{*}$, которые могут быть ускорены до зарегистрированных энергий в результате „кулоновского взрыва“, в зависимости от числа убегающих электронов $N_{e}^{(a c c)}$ и полное число ионов $N_{i \Sigma}$ в перетяжке на стадии после первого сжатия исходя из того, что радиус перетяжки и ее длина сравнимы по величине:

$$
N_{i \Sigma} \approx n_{i} \pi r^{3}
$$

$$
N_{i}^{*} \approx\left(e N_{e}^{(a c c)}\right)^{2} /\left(4 \pi \varepsilon_{0} r \varepsilon_{i}\right),
$$

где $n_{i} \approx 10^{25} \mathrm{~m}^{-3}-$ концентрация ионов [5,6]. Учитывая, что $N_{i}^{*} \leqslant N_{i \Sigma}$, наиболее близкий к реально регистрируемой величине $N_{i}$ результат дает значение $N_{e}^{(a c c)} \approx 10^{10}-10^{11}$.

Наша оценка количества высокоэнергетических ионов, основанная на измерении плотности потока ионов в радиальном направлении в предположении изотропности разлета, возможно, является заниженной. Существует поле резистивной природы, в котором ускоряются электроны:

$$
E_{\text {res }} \approx I R / r \sim 10^{8} \mathrm{~V} / \mathrm{m},
$$

где $R \approx 10^{-1} \Omega-$ сопротивление перетяжки, вычисленное с учетом потерь на излучение [20], $I \approx 10^{5} \mathrm{~A}-$ ток проводимости через перетяжку. Параметр, определяющий силовое воздействие со стороны магнитного поля на ион, оценим как

$$
E_{\text {mag }}=v_{i} B \approx\left(2 \varepsilon_{i} / M_{i}\right)^{1 / 2}\left[\mu_{0} I /(2 \pi r)\right] \sim 10^{9} \mathrm{~V} / \mathrm{m},
$$

где $B=\mu_{0} I /(2 \pi r)-$ индукция магнитного поля на границе перетяжки, $\mu_{0}-$ магнитная постоянная, $M_{i} \approx 10^{-25} \mathrm{~kg}$ - масса иона железа. Напряженность „кулоновского“ поля нескомпенсированного заряда ионов на стадии первого сжатия можно оценить как

$$
E_{c o l} \approx e N_{e}^{(a c c)} /\left(4 \pi \varepsilon_{0} r^{2}\right) \sim 10^{9}-10^{10} \mathrm{~V} / \mathrm{m}
$$

Следует учесть, что в процессе разлета плазмы из перетяжки вследствие „кулоновского“ взрыва воздействие „кулоновского“ поля на ионы убывает быстрее, чем воздействие магнитного поля.

Убегание электронов под действием электростатического электрического поля резистивной природы становится возможным в приосевой области, радиус которой приближается к величине ларморовского радиуса электрона на границе этой области [21]:

$$
r^{(a c c)}=r\left[2 \pi\left(2 m_{e} \varepsilon_{e}\right)^{1 / 2} /\left(\mu_{0} e I\right)\right]^{1 / 2} \approx 3 \cdot 10^{-6} \mathrm{~m},
$$

где $m_{e} \approx 10^{-30} \mathrm{~kg}$ - масса электрона, $\varepsilon_{e} \approx 50 \mathrm{eV}-$ средняя тепловая энергия электронов в перетяжке на стадии первого сжатия [3]. В этой же области должен был бы образоваться и избыточный положительный заряд, но тогда мы наблюдали бы среди регистрируемых высокоэнергетических ионов частицы с энергией, по крайней мере на порядок более высокой. Отсутствие таковых заставляет предположить следующий механизм формирования области плазмы с избыточным положительным зарядом. Нетрудно убедиться, что на стадии первого сжатия на границе перетяжки электроны и ионы замагничены. Следовательно, они дрейфуют вдоль оси разряда, обеспечивая протекание тока, что в силу значительного различия величины радиуса Лоренца для тех и других создает на внешней границе перетяжки тонкий (толщиной порядка радиуса Дебая) слой с избыточным положительным зарядом. Соответственно за пределами 
слоя плазма приобретает избыточный отрицательный заряд.

При включении механизма роста сопротивления плазмы в перетяжке происходит рост напряженности электрического поля, разогрев электронной компоненты плазмы и переход электронов в режим ускорения в приосевой области. В свою очередь произойдет увеличение радиуса Дебая и толщины внешнего слоя с избыточным положительным зарядом до величины:

$$
r_{\mathrm{D}}^{(*)} \approx\left[\left(2 \varepsilon_{0} \varepsilon_{e}^{(*)}\right) /\left(e^{2} n_{e}\right)\right]^{1 / 2} \approx 10^{-7} \mathrm{~m}
$$

где $\varepsilon_{e}^{(*)} \approx 10^{4} \mathrm{eV}$ - максимальная энергия ускоренных электронов, $n_{e} \approx 10^{26} \mathrm{~m}^{-3}$ [22,23]. Количество ионов в дебаевском слое будет составлять порядка $10^{9}-10^{10}$.

С переходом электронов в приосевой области перетяжки в режим непрерывного ускорения будет происходить обеднение электронами плазмы за пределами дебаевского слоя, и она будет приближаться к квазинейтральному состоянию, а ионы в дебаевском слое испытают „кулоновский взрыв“. Продолжительность ухода убегающих электронов из перетяжки и продолжительность „кулоновского взрыва“ оценим как

$$
\begin{gathered}
\tau_{f l y}^{(*)} \approx r /\left(2 \varepsilon_{e}^{(*)} / m_{e}\right)^{1 / 2} \approx 10^{-12} \mathrm{~s}, \\
\tau_{\text {expl }} \approx r /\left(2 \varepsilon_{i} / M_{i}\right)^{1 / 2} \approx 3 \cdot 10^{-11} \mathrm{~s} .
\end{gathered}
$$

Скорость ускоренных электронов, покидающих перетяжку, более чем на порядок превышает тепловую скорость электронов, поступающих в перетяжку. Поэтому образующийся в результате явления убегания дефицит количества электронов в перетяжке оценим следующим образом:

$$
\begin{aligned}
\Delta N_{e} & \approx \pi\left(r^{(a c c)}\right)^{2} r n_{e}-\left[n_{e}\left(2 \varepsilon_{e} / m_{e}\right)^{1 / 2} / 4\right] \\
& \times \pi\left(r^{(a c c)}\right)^{2} \tau_{\text {expl }} \approx 10^{10}-10^{11}
\end{aligned}
$$

Таким образом, можно говорить о том, что экспериментально обнаружены свидетельства возможной реализации „кулоновского взрыва“ дебаевского слоя в области формирования микропинча в разряде типа $Z$-пинч в среде тяжелых элементов вследствие убегания ускоренных в осевом направлении высокоэнергетических электронов.

\section{Список литературы}

[1] Орешкин В.И., Орешкин Е.В. // ЖТФ. 2017. Т. 87. В. 1. C. 34-38.

[2] Долгов А.Н., Клячин Н.А., Прохорович Д.Е. // ЖТФ. 2014. T. 84. B. 12. C. $145-148$.

[3] Вихрев В.В., Иванов В.В., Кочелев К.Н. // Физика плазмы. 1982. T. 8. № 6. C. 1211-1219.

[4] Веретенников В.А., Долгов А.Н., Крохин О.Н., Семёнов О.Г. // Физика плазмы. 1985. Т. 11. № 8. С. 1107-1110.

[5] Филиппов Н.В. // ЖЭТФ. 1979. Т. 76. В. 5. С. 1547-1550.
[6] Nishio M., Sakuma H., Tarasugi K. // Plasma Fusion Res. 2011. V. 6. P. 1201009.

[7] Плютто А.А. // ЖЭТФ. 1960. Т. 39. В. 6. С. 1589-1592.

[8] Pease R.S. // Proc. Phys. Soc. B (London). 1957. V. 70. N 1. P. 11-23.

[9] Брагинский С.И. // ЖЭТФ. 1957. Т. 33. В. 3. С. 645-654.

[10] Spearer J.W. // Phys. Fluids. 1976. V. 19. N 9. Р. 1426-1427.

[11] Блинников С.И., Имшенник В.С. // Физика плазмы. 1982. T. 8. № 1. С. 193-205.

[12] Орешкин В.И. // Изв. вузов. Физика. 1997. Т. 40. № 12. C. $76-83$.

[13] Бакшаев Ю.Л., Блинов П.И., Вихрев В.В., Гордеев Е.М., Данько С.А., Королев В.Д., Медовщиков С.Ф., Недосеев С.Л., Смирнова Е.А., Туманов В.И., Черненко А.С., Шашков А.Ю. // Физика плазмы. 2001. Т. 27. № 12. C. 1101-1109.

[14] Bernal L., Bruzzone H. // Plasma Phys. Contr. Fusion. 2002. V. 44. N 2. P. 223-231.

[15] Дубинов А.Е., Корнилова И.Ю., Селемир В.Д. // УФН. 2002. T. 172. № 11. С. 1225-1246.

[16] Рухадзе А.А., Юсупалиев У. // ЖТФ. 2004. Т. 74. В. 7. C. $127-128$.

[17] Иванников Г.В., Мингалеев А.Р., Пикуз С.А., Романов В.М., Шелковенко Т.А. // Физика плазмы. 1996. Т. 22. № 5. C. 403-418.

[18] Pikuz S.A., Shelkovenko T.A., Sinars D.B., Greenly J.B., Dimant Y.S., Hammer D.A. // Phys. Rev. Lett. 1999. V. 83. N 21. P. 4313-4316.

[19] Sinars D.B., Hu M., Chandler K.M., Shelkovenko T.A., Pikuz S.A., Greenly J.B., Hammer D.A., Kusse B.R. // Phys. Plasmas. 2001. V. 8. N 1. P. 216-230.

[20] Долгов А.Н., Вихрев В.В. // Физика плазмы. 2005. Т. 31. № 3. C. $290-297$.

[21] Долгов А.Н., Земченкова Н.В., Клячин Н.А., Прохорович Д.Е. // Физика плазмы. 2011. Т. 37. № 3. С. 227-232.

[22] Саркисов Г.С., Этлишер Б. // Письма в ЖЭТФ. 1995. Т. 62. B. 9-10. C. 775-782.

[23] Веретенников В.А., Гурей А.Е., Писарчик Т., Полухин С.Н., Рупасов А.А., Саркисов Г.С., Семёнов О.Г., Шиканов А.С. // Физика плазмы. 1990. Т. 16. № 7. С. 818 822. 